\title{
Exploring the barriers and facilitators to the consumption of eggs and other protein rich foods using focus groups
}

\author{
E. van den Heuvel ${ }^{1}$, J.L. Murphy ${ }^{2}$ and K.M. Appleton ${ }^{1}$ \\ ${ }^{1}$ Department of Psychology, Bournemouth University, Poole BH12 5BB and ${ }^{2}$ School of Health and Social Care, \\ Bournemouth University, Bournemouth BHI 3 LT
}

Sarcopenia is the age-related loss of skeletal muscle mass and strength, and it has been suggested that inadequate dietary protein intake might be one of the risk factors ${ }^{(1)}$. Previous research exploring the factors associated with the consumption of protein-rich foods in older adults suggests difficulties particularly based on the texture, perishability and need-to-cook nature of most high protein foods ${ }^{(2)}$. In comparison to other protein-rich foods, however, eggs are of soft texture, long shelf-life, relatively low cost, and are easy to $\operatorname{cook}^{(3,4)}$, and so may offer a solution. This study explored the barriers and facilitators to the consumption of eggs and other proteinrich foods using focus groups and interviews. With an interest in prevention as well as treatment, the sample ranged from aged 55 years upwards.

A total of 20 males and 19 females took part in one of 6 focus groups or two interviews. Participants were independently living individuals, ranging in age from 56 to 84 years, of whom 11 were employed (working over 20 hours per week), and 7 were classified as frail (self-reported to meet the criteria set by Fried et al. ${ }^{(5)}$ ). Focus groups included gender-specific groups, and separate groups for those working and those who do not work. Participants were asked to discuss the reasons why they do or do not eat eggs, dairy products, meat, fish, seafood, nuts and pulses, whilst being shown images of each type of food. Conversations lasted for about one hour, and were audio recorded, transcribed, and analysed using thematic analysis by two researchers independently. Analyses focussed exclusively on references to eggs or protein-rich foods: comments about eating behaviour in general were not included.

Agreed barriers and facilitators to consumption were related to: hedonics (e.g. liking); the properties of the food (e.g. taste, texture); preparation style (e.g. part of a dish); convenience (e.g. time, effort to cook); physical environment (e.g. financial difficulties, availability); variety (e.g. replacing other foods); physical health/ability (e.g. biting, chewing and swallowing difficulties, medical constraints); nutrition and health knowledge (e.g. health beliefs, nutrition and health advice); food safety (e.g. food scares); social environment (e.g. others in the house, or culture); morality (e.g. animal welfare); emotions (e.g. treat); and habit (e.g. upbringing).

Many of these reasons for consuming / not consuming eggs and other protein-rich foods are well known factors associated with food consumption, but certain factors may be particularly important in determining egg consumption and for the ageing population. The determinants identified in this study will now be used to design a questionnaire to explore the relative importance of each factor, in relation to protein intake, in a large representative sample. A more thorough understanding of the reasons for eating these specific foods in this population will aid the design of practical interventions to increase dietary protein intake and improve protein status in this population group.

1. Cruz-Jentoft AJ, Baeyens JP, Bauer JM, et al. (2010) Age Ageing 39, 412-423.

2. Best RL \& Appleton KM (2013) J Nutr Educ Behav 45, 751-755.

3. Drewnowski A (2010) Am J Clin Nutr 91, 1095-1101.

4. Lewis BR \& Bashin A (1988) Brit Food J 90, 172-177.

5. Fried LP, Tangen CM, Walston J, et al. (2001) J Gerontol A Biol Sci Med Sci 56, 146-156. 